\title{
EVEROLIMO E SEU PAPEL POTENCIAL NO TRANSPLANTE RENAL
}

\author{
The potential role of everolimus after kidney transplantation
}

Helio Tedesco-Silva Jr', Glaucia Prismich²

\section{RESUMO}

As taxas de sobrevida do enxerto ainda são baixas em longo prazo, pois o uso de alguns dos novos agentes imunossupressores, que muito contribuíram para a significativa diminuição das taxas de rejeição aguda, não impede o aparecimento e a evolução da disfunção crônica do enxerto. Com o objetivo de fazer uma revisão sobre o uso do everolimo na imunossupressão de pacientes receptores de transplante renal e seu potencial uso na prevenção da disfunção progressiva do enxerto, foi realizada uma busca por artigos nas bases de dados PUBMED E LILACS (Literatura Latino-Americana e do Caribe em Ciências da Saúde) entre 1997 e 2007, e foram considerados artigos elegíveis aqueles que compararam o uso do everolimo com micofenolato de mofetila ou com azatioprina no tratamento imunossupressor de pacientes submetidos a transplantes de órgãos, assim como artigos que discorriam sobre transplante renal e suas complicações e sobre o uso do everolimo. A disfunção crônica do enxerto, cuja prevalência comprovada por biópsia pode chegar a $94 \%$ em um ano, tem alguns fatores de risco como: episódios de rejeição aguda; infecção por citomegalovírus; nefrotoxicidade induzida pelo uso de inibidores da calcineurina; e comorbidades como diabetes mellitus, hipertensão arterial e dislipidemia. O everolimo, um potente imunossupressor, que atua como inibidor do sinal de proliferação celular e tem uso potencial na prevenção da disfunção crônica do enxerto, quando associado à ciclosporina.

Descritores: Falência Renal Crônica; Imunossupressores; Transplante de Rim.

\section{Instituições:}

${ }^{1}$ Setor de Transplante Renal, Hospital do Rim Hipertensão/ UNIFESP

${ }^{2}$ Novartis Biociências S.A.

\section{Correspondência:}

Helio Tedesco-Silva, MD,

Rua Borges Lagoa, 960 - 110 andar 04038-002 - São Paulo, SP, Brasil

Tel.: 551150878113

Fax: 551150878145

Email: heliotedesco@hrim.com.br

Recebido em: 17.09 .2006

\section{INTRODUÇÃO}

Em 1965, foi realizado no Brasil o primeiro transplante renal com sucesso. ${ }^{1}$ Hoje, cerca de 3.200 pacientes por ano são submetidos a transplante renal no Brasil. ${ }^{2}$ Essa evolução só foi alcançada devido à melhora das técnicas cirúrgicas e ao advento de novos regimes de terapia imunossupressora. Atualmente, a sobrevida do paciente receptor de transplante renal é de $94 \%$ em um ano, $88 \%$ após três anos e mais de $79 \%$ após cinco anos; esses índices são devidos, em grande parte, ao aprimoramento da terapia imunossupressora. ${ }^{3}$ Entretanto, as taxas de sobrevida do enxerto ainda são baixas no longo prazo, pois o uso de alguns dos novos agentes imunossupressores, que muito contribuíram para a significativa diminuição da taxa de rejeição aguda, não impede o aparecimento e a evolução de disfunção crônica do enxerto, muitas vezes denominada "rejeição crônica". Atualmente, a rejeição aguda não constitui uma causa freqüente de perda do enxerto, porém, contribui para a disfunção crônica do enxerto, principal causa de perda do enxerto renal.

\section{Disfunção Crônica do Enxerto}

A disfunção crônica do enxerto, cuja prevalência comprovada por biópsia pode chegar a $94 \%$ em um ano, tem como fatores de risco: episódios de rejeição aguda, infecção por citomegalovírus (CMV), nefrotoxicidade induzida pelo uso de inibidores da calcineurina e comorbidades como diabetes mellitus, hipertensão arterial e dislipidemia. ${ }^{4}$ 
A relação causal entre infecção pelo CMV e disfunção crônica do enxerto ainda não é bem estabelecida. ${ }^{5} \mathrm{~A}$ infecção por CMV está mais associada à rejeição aguda, já que aparece nos primeiros seis meses após o transplante.

Além de provocar lesões teciduais e doenças agudas causadas pela toxicidade do vírus, ${ }^{6}$ a infecção pelo CMV também determina um aumento do grau de imunossupressão e aumenta o risco de outras infecções oportunistas, como a infecção fúngica invasiva. Acredita-se que esta seja sua principal contribuição como causa da disfunção do enxerto. ${ }^{6}$

Os inibidores de calcineurina, como a ciclosporina (CsA) e o tacrolimo são medicamentos indispensáveis na terapia imunossupressora de paciente receptor de transplante renal, já que seu uso está associado a um aumento na sobrevida do enxerto e, conseqüentemente, na sobrevida do paciente. ${ }^{7}$ Porém, a nefrotoxicidade associada a esses medicamentos ainda é uma causa importante de perda do enxerto. ${ }^{7}$ A redução da dose a ser administrada a pacientes transplantados renais com evidência histológica de nefrotoxicidade tem sido adotada como alternativa eficaz na melhora da função renal. ${ }^{8}$ Outra forma de evitar a nefrotoxicidade dos inibidores de calcineurina é adicionar novas drogas aos tradicionais regimes imunossupressores, com o intuito de diminuir a dose dos inibidores de calcineurina, sem que ocorra, assim, um aumento do risco de rejeição aguda.

O everolimo, um potente imunossupressor que atua como inibidor do sinal de proliferação celular, tem potencial uso na prevenção da disfunção crônica progressiva do enxerto, quando associado à CsA, pois permite que as doses do inibidor de calcineurina sejam reduzidas com segurança, já que em associação, o everolimo e a CsA atuam de forma sinérgica. ${ }^{9-11} \mathrm{O}$ presente artigo faz uma revisão sobre o uso do everolimo na imunossupressão de pacientes receptores de transplante renal e seu potencial uso na prevenção da disfunção crônica progressiva do enxerto.

\section{MÉTODOS}

Foi realizada uma busca inicial por artigos nas bases de dados PUBMED E LILACS (Literatura Latino-Americana e do Caribe em Ciências da Saúde) entre 1997 e 2007. Outros artigos relevantes foram identificados a partir da revisão das listas de referência dos artigos selecionados na busca inicial. Foram considerados artigos elegíveis aqueles que compararam o uso do everolimo com micofenolato de mofetila ou com azatioprina no tratamento imunossupressor de pacientes submetidos a transplantes de órgãos, assim como artigos que discorriam sobre transplante renal e suas complicações, e sobre o uso do everolimo. Não foi feita restrição em relação ao idioma do artigo.

\section{RESULTADOS E DISCUSSÃO}

\section{Everolimo: Farmacocinética}

O everolimo é uma droga semi-sintética, derivada da rapamicina (um macrolídeo produzido pelo Streptomyces hygroscopicus), que atua como inibidor do sinal de proliferação celular. ${ }^{12}$ Administrado por via oral, o everolimo é rapidamente absorvido, atingindo uma concentração sérica máxima em uma a duas horas após sua administração e alcançando o estado de equilíbrio dentro de quatro dias. ${ }^{13-14} \mathrm{O}$ everolimo, que é metabolizado por isoenzimas do citocromo $\mathrm{P} 450,{ }^{15}$ apresenta farmacocinética proporcional à dose, e a monitorização terapêutica de seu nível sangüíneo pode otimizar sua eficácia e segurança. Estudos da fase III identificaram o nível sangüíneo mínimo eficaz do everolimo como sendo de três ng/ml. ${ }^{16}$ Além disso, dois estudos prospectivos que avaliaram a ação do everolimo nas doses de 1,5 e 3,0 mg/dia, associado a CsA e corticóide, e ao basiliximabe como terapia imunossupressora em pacientes receptores de transplante renal, num dos estudos, chegaram à conclusão de que quando o everolimo é mantido abaixo da concentração mínima de $3 \mathrm{ng} / \mathrm{ml}$, a taxa de rejeição aguda comprovada por biópsia é significativamente maior, como demonstra a Figura $1 .{ }^{17}$

Figura 1: Incidência de rejeição aguda comprovada por biópsia em 6 meses, de acordo com a concentração sérica de everolimo. ${ }^{17}$

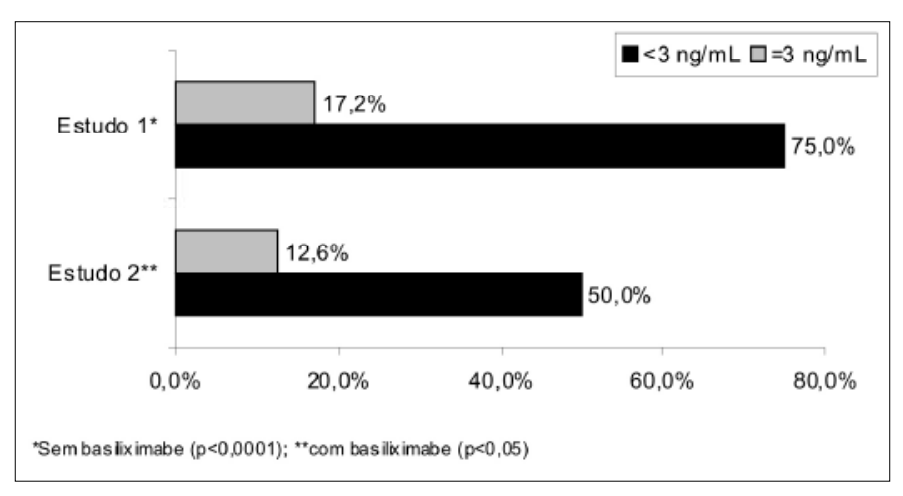

Tabela 1: Resultados de eficácia com 12 meses dos principais estudos com everolimo. ${ }^{26-28}$

\begin{tabular}{|c|c|c|c|}
\hline 12 meses & RACB & Perda do enxerto & Óbito \\
\hline \multicolumn{4}{|l|}{$\mathrm{B} 201^{27}$} \\
\hline Everolimo 1,5mg/d & $23,2 \%$ & $4,6 \%$ & $5,2 \%$ \\
\hline Everolimo $3,0 \mathrm{mg} / \mathrm{d}$ & $19,7 \%$ & $10,6 \%$ & $4,0 \%$ \\
\hline \multicolumn{4}{|l|}{$B 251^{26}$} \\
\hline Everolimo 1,5mg/d & $19,2 \%$ & $8,8 \%$ & $3,1 \%$ \\
\hline Everolimo $3,0 \mathrm{mg} / \mathrm{d}$ & $22,2 \%$ & $4,1 \%$ & $3,6 \%$ \\
\hline MMF & $24,0 \%$ & $5,1 \%$ & $2,0 \%$ \\
\hline \multicolumn{4}{|l|}{ RAD2306 31} \\
\hline Everolimo 1,5mg/d & $25,9 \%$ & $5,4 \%$ & $0,9 \%$ \\
\hline Everolimo $3,0 \mathrm{mg} / \mathrm{d}$ & $19,2 \%$ & $3,2 \%$ & $4,8 \%$ \\
\hline \multicolumn{4}{|l|}{ RAD230731 } \\
\hline Everolimo $1,5 \mathrm{mg} / \mathrm{d}$ & $13,7 \%$ & $1,7 \%$ & 0 \\
\hline Everolimo $3,0 \mathrm{mg} / \mathrm{d}$ & $15,8 \%$ & $5,0 \%$ & $1,4 \%$ \\
\hline
\end{tabular}

\section{Efeito Imunossupressor do Everolimo}

O everolimo inibe a ação da enzima fosfatidilinositol-3-quinase e, conseqüentemente, a proliferação celular das seguintes células: linfócitos $\mathrm{B}$, linfócitos $\mathrm{T}$, fibroblastos e músculo liso vascular. Essa inibição é devida ao efeito bloqueador do everolimo sobre 
Tabela 2: Resultados da função renal com 12 meses dos principais estudos com everolimo. ${ }^{26-28}$

\begin{tabular}{|c|c|c|}
\hline 12 meses & $\begin{array}{l}\text { Creatinina sérica } \\
\text { média }(\mu \mathrm{mol} / \mathrm{L})\end{array}$ & $\begin{array}{c}\text { Clearance de creatinina } \\
\text { média ( } \mathrm{ml} / \mathrm{min})\end{array}$ \\
\hline \multicolumn{3}{|l|}{ B20127 } \\
\hline Everolimo 1,5mg/d & 175 & 52 \\
\hline Everolimo $3,0 \mathrm{mg} / \mathrm{d}$ & 216 & 47 \\
\hline \multicolumn{3}{|l|}{$B 251^{26}$} \\
\hline Everolimo 1,5mg/d & 150 & 58 \\
\hline Everolimo $3,0 \mathrm{mg} / \mathrm{d}$ & 159 & 52 \\
\hline MMF & 133 & 69 \\
\hline \multicolumn{3}{|l|}{ RAD2306 31} \\
\hline Everolimo 1,5mg/d & 131 & 65 \\
\hline Everolimo $3,0 \mathrm{mg} / \mathrm{d}$ & 130 & 63 \\
\hline \multicolumn{3}{|l|}{ RAD2307 31} \\
\hline Everolimo 1,5mg/d & 129 & 64 \\
\hline Everolimo $3,0 \mathrm{mg} / \mathrm{d}$ & 128 & 64 \\
\hline
\end{tabular}

a transdução dos sinais gerados pelas interleucinas (IL) 2 e IL-15 em células hematopoiéticas e não-hematopoiéticas. ${ }^{18} \mathrm{O}$ everolimo possui, portanto, um mecanismo imunossupressor totalmente diferente dos inibidores de calcineurina, já que estes últimos promovem diretamente a inativação dos linfócitos T. Acreditase que a associação de diferentes mecanismos de ação resulte na ação sinérgica dos dois medicamentos, como demonstraram estudos pré-clínicos realizados em modelos animais. ${ }^{10,18}$ Baseados nestes achados, alguns autores afirmam que o everolimo permite a diminuição da exposição à CsA sem aumentar o risco de rejeição, o que reduz potencialmente os eventos adversos, como a nefrotoxicidade induzida pelos inibidores da calcineurina, fator determinante na disfunção crônica do enxerto. ${ }^{5,11,19}$

\section{Efeitos Antiproliferativos}

A disfunção crônica do enxerto é freqüentemente caracterizada pela proliferação das células do músculo liso da camada íntima dos vasos do enxerto e por aterosclerose. Estudos experimentais indicam que ao inibir a ação de imunomediadores inflamatórios e a proliferação de fibroblastos e de células musculares lisas vasculares, o everolimo previne o remodelamento vascular, evitando assim o espessamento da camada íntima dos vasos do enxerto. ${ }^{11,20}$

\section{Eventos Adversos}

Os inibidores do sinal de proliferação celular têm como um dos principais eventos adversos a hiperlipidemia, caracterizada pelo aumento do nível sérico de colesterol e triglicérides, que afeta entre $30 \%$ e $50 \%$ dos pacientes tratados. ${ }^{21} \mathrm{Um}$ estudo experimental conduzido em camundongos, com o intuito de investigar a hiperlipidemia induzida pelo everolimo, demonstrou que o medicamento pode alterar a homeostase lipídica celular nos macrófagos peritoneais de camundongos, quando administrado em uma concentração próxima à dose terapêutica $(0,01 \mu \mathrm{M}), \mathrm{o}$ que resulta no aumento da esterificação e do efluxo do colesterol, assim como na redução da biossíntese dos triglicérides. ${ }^{2}$ Em geral, recomenda-se o tratamento da dislipidemia com uma combinação de estatinas para reduzir o colesterol sérico, e fibratos para reduzir os triglicérides séricos, bem como a adoção de um estilo de vida saudável..$^{21}$ Estudos experimentais recentes têm demonstrado que a associação das estatinas ao tratamento com o everolimo pode trazer ainda mais benefícios.

Demonstrou-se que o uso combinado de everolimo e fluvastatina resultou numa ação anti-proliferativa 8,4 vezes maior nas células musculares lisas da camada íntima da aorta de camundongos, quando comparado com o uso isolado do everolimo. ${ }^{22}$

Dois estudos prospectivos randomizados foram conduzidos em aproximadamente 490 pacientes submetidos a transplante renal com o objetivo de avaliar a terapia com everolimo nas doses de 1,5 ou 3,0 mg/dia, CsA em dose reduzida e corticosteróides. Em um desses estudos, o tratamento também incluiu basiliximabe. Os estudos mostraram que a maioria dos pacientes apresentou algum evento adverso relacionado a esses regimes terapêuticos. $\mathrm{O}$ evento adverso de maior incidência foi infecção em cerca de $50 \%$ a $60 \%$ dos pacientes, sendo a infecção do trato urinário a mais comum em $20 \%$ a $30 \%$ dos casos. ${ }^{17}$ Com relação à toxicidade hematológica, a anemia microcítica foi encontrada em cerca de $20 \%$ dos pacientes que receberam everolimo; porém, a incidência de anemia é praticamente igual, quando comparados os inibidores de proliferação celular com o micofenolato de mofetila. ${ }^{17,23}$ Nesses estudos randomizados, a anemia foi tratada conforme sua gravidade. Na maioria dos casos em que foi necessário tratamento, a dose do everolimo foi reduzida. Em casos mais graves, além de reduzir a dose de everolimo, os investigadores optaram pela introdução de eritropoietina. Em um único caso isolado, o everolimo foi descontinuado devido a importante trombocitopenia. ${ }^{17}$

Linfocele também foi um evento adverso comumente relatado nos estudos realizados com everolimo. Nos estudos randomizados, a incidência de linfocele foi de $6,4 \%$ a $15,2 \%$, sendo que as incidências mais altas foram encontradas nos grupos de pacientes que receberam everolimo em doses menores ( $1,5 \mathrm{mg} / \mathrm{dia}) .{ }^{17}$

A linfocele é uma complicação não raramente encontrada em pacientes submetidos a transplantes renais, principalmente nas primeiras semanas após o transplante; entretanto, nos estudos que envolvem o uso de inibidores de proliferação celular, como o everolimo e o sirolimo, essa incidência tem sido um pouco maior que o esperado..$^{21,24,25} \mathrm{Na}$ grande maioria dos casos relatados, a linfocele se resolveu espontaneamente ou foi facilmente abordada através da drenagem e instilação de solução iodada. Houve relato de um único caso no qual foi necessária abordagem cirúrgica laparoscópica. ${ }^{25} \mathrm{Em}$ nenhum dos estudos foi necessária diminuição da dose a ou a descontinuação do medicamento devido à linfocele..$^{21,24,25} \mathrm{O}$ único evento adverso relatado por mais de dois pacientes e que levou à descontinuação do everolimo foi a artralgia. ${ }^{17}$

\section{Eficácia Clínica do Everolimo}

Os estudos prospectivos randomizados que avaliaram a combinação de everolimo nas doses de 1,5 ou $3 \mathrm{mg} / \mathrm{dia}$, corticóide e CsA em 
dose reduzida, associada ou não a basiliximabe demonstraram que é possível obter uma imunossupressão adequada, desde que a dose sérica de everolimo seja adequadamente monitorada (Figura 1). ${ }^{17} \mathrm{O}$ everolimo, quando mantido em nível sangüíneo maior que $3 \mathrm{ng} / \mathrm{ml}$, permitiu que o paciente fosse exposto a uma menor dose de CsA, havendo assim diminuição da potencial nefrotoxicidade causada pela droga, sem que houvesse aumento da taxa de rejeição aguda do enxerto ou prejuízo da função renal. Mais de $50 \%$ dos pacientes mantiveram o nível de creatinina sérica menor ou igual a $1,5 \mathrm{mg} / \mathrm{dL}$ nos primeiros seis meses pós-transplante, o que determina um bom prognóstico em relação à sobrevida do enxerto em longo prazo.

Menos de $15 \%$ dos pacientes apresentaram nível sérico de creatinina superior a $2,1 \mathrm{mg} / \mathrm{dL}$, que está associado a uma baixa taxa de sobrevida do enxerto. ${ }^{17}$ Estudos demonstraram que o everolimo é tão eficaz quanto o micofenolato de mofetila na prevenção da rejeição aguda, sem haver piora da função renal. ${ }^{17,26,27}$

Os principais resultados de eficácia com 12 meses estão resumidos na tabela $1 .{ }^{26-28}$

Os resultados da função renal com 12 meses encontram-se na tabela $2 . .^{26-28}$

A incidência de falha de eficácia (rejeição aguda comprovada por biópsia, perda do enxerto, óbito ou perda de seguimento) com 36 meses em dois estudos multicêntricos randomizados comparando duas doses diferentes de everolimo $(1,5 \mathrm{mg} / \mathrm{d}$ e $3,0 \mathrm{mg} / \mathrm{d}$ ) e doses reduzidas de ciclosporina com ou sem indução com basiliximabe foi: $31 \%$ e $32 \%$ no estudo sem indução, e $25 \%$ e $26 \%$, no estudo com indução, para as doses de $1,5 \mathrm{mg} / \mathrm{d}$ e 3,0 $\mathrm{mg} / \mathrm{d}$, respectivamente $(\mathrm{p}=\mathrm{NS}){ }^{29,30}$

Em relação à incidência de infecção por CMV, um estudo randomizado da fase III envolvendo 600 pacientes submetidos a transplante cardíaco e que receberam everolimo ou azatioprina como terapia imunossupressora associados à CsA em dose plena constatou que houve menor taxa de infecção por CMV no grupo que recebeu everolimo (7,7\% versus $21,5 \% ; \mathrm{p}<0,001) .{ }^{31}$ Além disso, nesse mesmo estudo foi constatado que pacientes que receberam everolimo apresentaram espessamento da camada íntima dos vasos do enxerto significativamente menor que pacientes que receberam azatioprina, e, conseqüentemente, a incidência de vasculopatia do enxerto foi menor no grupo que recebeu everolimo (37,5\% versus $52,8 \%$; $=0,045) .{ }^{31}$ Outro estudo prospectivo e randomizado conduzido em 588 pacientes submetidos a transplante renal comparando o everolimo nas doses de 1,5 e $3 \mathrm{mg} / \mathrm{dia}$, com micofenolato de mofetila na dose de $2 \mathrm{~g} /$ dia constatou que houve menor incidência de infecções virais particularmente por CMV nos grupos que receberam everolimo. ${ }^{27}$

Do ponto de vista fármaco-econômico, os estudos que compararam o uso do everolimo nas doses de 1,5 e $3 \mathrm{mg} /$ dia ao uso de azatioprina em pacientes que receberam transplante cardíaco e ao uso de micofenolato de mofetila em pacientes que receberam transplante renal demonstraram que não houve incremento dos custos totais do tratamento imunossupressor. ${ }^{32,33}$

\section{CONCLUSÃO}

Com base nos dados acima mencionados, pode-se concluir que o everolimo é um medicamento eficaz na prevenção da rejeição aguda em pacientes transplantados renais, quando comparado aos medicamentos atualmente usados, entre eles o micofenolato de mofetila e a azatioprina. $\mathrm{O}$ everolimo permite que os pacientes sejam expostos a menor dose de CsA, sem que haja redução da eficácia da imunossupressão, diminuindo assim a chance de uma potencial nefrotoxicidade. Além disso, tem sido relatada menor incidência de infecção por CMV em pacientes que receberam everolimo, bem como uma importante ação do everolimo na prevenção da vasculopatia do enxerto, já que sob seu efeito, ocorre menor espessamento da camada íntima dos vasos. Desta maneira, acredita-se que o everolimo poderia determinar uma menor incidência da disfunção progressiva do enxerto, principal causa de perda do enxerto, já que ele diminui a incidência dos principais fatores envolvidos em sua gênese (vasculopatia, rejeição aguda e infecção por CMV). ${ }^{34}$

Porém, como o everolimo ainda é uma droga nova, são necessários mais estudos de longo prazo para que se possa avaliar de forma adequada seus benefícios na prevenção da disfunção progressiva do enxerto.

\section{ABSTRACT}

The long-term graft survival rates are still relatively low. The use of some novel immunosuppressant, which played a significant role in reducing the risk of acute rejection, does not prevent the development and progression of chronic allograft dysfunction. The aim of this article is to provide a review on the use of everolimus as immunosuppressive therapy for kidney transplant recipients, and on its potential role in preventing chronic allograft nephropathy.

Articles published on Pubmed and Lilacs between 1997 and 2007, were considered for this review and were eligible those comparing the use of everolimus versus mycophenolate mofetil or azathioprine in transplant recipients and also articles that focused renal transplant complications.

Chronic allograft nephropathy, whose biopsy proven prevalence may be as high as $94 \%$ in the first post-transplantation year has some risk factors such as: episodes of acute rejection; cytomegalovirus infection; nephrotoxicity induced by calcineurin inhibitors and comorbidities, such as diabetes mellitus, hypertension and dyslipidemia. Everolimus, a potent immunosuppressant that works as an inhibitor of cellular proliferation has a potential efficacy in preventing chronic allograft dysfunction, when combined with cyclosporine.

Keywords: Kidney Failure, Chronic; Immunosuppressive Agents; Kidney Transplantation 


\section{REFERÊNCIAS}

1. Salomão A. Atualização em Transplante Renal. J Bras Nephrol. 2000;22:244-8

2. ABTO (Associação Brasileira de Transplantes de Orgãos), 2006. Disponível em http://www.abto.org.br. (Acesso em 20/10/2006)

3. Hariharan S, Johnson CP, Bresnahan BA, Taranto SE, McIntosh MJ, Stablein D. Improved graft survival after renal transplantation in the United States, 1988 to 1996. N Engl J Med. 2000;342:605-12

4. Nankivell BJ, Borrows RJ, Fung CL, O'Connell PJ, Allen RD, Chapman JR. The natural history of chronic allograft nephropathy. N Engl J Med. 2003;349:2326-33

5. Johnson RW. The clinical impact of nephrotoxicity in renal transplantation. Transplantation. 2000;69(12 Suppl):SS14-7

6. Tong CY, Bakran A, Peiris JS, Muir P, Herrington CS. The association of viral infection and chronic allograft nephropathy with graft dysfunction after renal transplantation. Transplantation. 2002;74:576-8

7. Ponticelli C. Clinical experience with everolimus (Certican): a summary. Transplantation. 2005;79(9 Suppl):S93-4

8. Pilmore HL, Dittmer ID. Calcineurin inhibitor nephrotoxicity: reduction in dose results in marked improvement in renal function in patients with coexisting chronic allograft nephropathy. Clin Transplant. 2002;16:191-5

9. Johnson S, Qi S, Xu D, Jolicoeur M, Liu D, Barama A, et al. Synergistic effects of RAD and Neoral in inhibition of host-vs.-graft and graft-vs.-host immune responses in rat small-bowel transplantation. Microsurgery. 2003;23:476-82

10. Schuurman HJ, Ringers J, Schuler W, Slingerland W, Jonker M. Oral efficacy of the macrolide immunosuppressant SDZ RAD and of cyclosporine microemulsion in cynomolgus monkey kidney allotransplantation. Transplantation. 2000;69:737-42

11. Neumayer HH. Introducing everolimus (Certican) in organ transplantation: an overview of preclinical and early clinical developments. Transplantation. 2005;79(9 Suppl):S72-5

12. Formica RN Jr, Lorber KM, Friedman AL, Bia MJ, Lakkis F, Smith JD, et al. The evolving experience using everolimus in clinical transplantation. Transplant Proc. 2004;36(2 Suppl):495S-9S

13. Kovarik JM, Kahan BD, Kaplan B, Lorber M, Winkler M, Rouilly M, et al. Longitudinal assessment of everolimus in de novo renal transplant recipients over the first post-transplant year: pharmacokinetics, exposure-response relationships, and influence on cyclosporine. Clin Pharmacol Ther. 2001;69:48-56

14. Kovarik JM, Noe A, Berthier S, McMahon L, Langholff WK, Marion AS, et al. Pharmacokinetics of an everolimus-cyclosporine immunosuppressive regimen over the first 6 months after kidney transplantation. Am J Transplant. 2003;3:606-13

15. Kirchner GI, Winkler M, Mueller L, Vidal C, Jacobsen W, Franzke A, et al. Pharmacokinetics of SDZ RAD and cyclosporin including their metabolites in seven kidney graft patients after the first dose of SDZ RAD. Br J Clin Pharmacol. 2000;50:449-54

16. Lorber MI, Ponticelli C, Whelchel J, Mayer HW, Kovarik J, Li Y, et al. Therapeutic drug monitoring for everolimus in kidney transplantation using 12-month exposure, efficacy, and safety data. Clin Transplant. 2005;19:145-52

17. Vitko S, Tedesco H, Eris J, Pascual J, Whelchel J, Magee JC, et al. Everolimus with optimized cyclosporine dosing in renal transplant recipients: 6-month safety and efficacy results of two randomized studies. Am J Transplant. 2004;4:626-35
18. Schuler W, Sedrani R, Cottens S, Haberlin B, Schulz M, Schuurman HJ, et al. SDZ $\mathrm{RAD}$, a new rapamycin derivative: pharmacological properties in vitro and in vivo. Transplantation. 1997;64:36-42

19. Meier-Kriesche HU, Ojo AO, Hanson JA, Cibrik DM, Punch JD, Leichtman AB, et al. Increased impact of acute rejection on chronic allograft failure in recent era. Transplantation. 2000;70:1098-100

20. Cole OJ, Shehata M, Rigg KM. Effect of SDZ RAD on transplant arteriosclerosis in the rat aortic model. Transplant Proc. 1998;30:2200-3

21. Pascual J. Everolimus in clinical practice-renal transplantation. In: Nephrol Dial Transplant. 2006:iii18-23

22. Ferri N, Granata A, Pirola C, Pfister PJ, Dorent R, Corsini A. In vitro enhanced antiproliferative activity of everolimus by fluvastatin on arterial smooth muscle cells. International Symposium on Atheroesclerosis, Rome, Italy, June 18-22, 2006.

23. Kahan BD. Efficacy of sirolimus compared with azathioprine for reduction of acute renal allograft rejection: a randomised multicentre study. The Rapamune US Study Group. Lancet. 2000;356:194-202

24. Pascual J, Marcen R, Ortuno J. Clinical experience with everolimus (Certican): optimizing dose and tolerability. Transplantation 2005;79(9 Suppl):S80-4

25. Eris J. Clinical experience with everolimus (Certican) in young renal transplant recipients. Transplantation. 2005;79(9 Suppl):S89-92

26. Lorber MI, Mulgaonkar S, Butt KM, Elkhammas E, Mendez R, Rajagopalan PR, et al. Everolimus versus mycophenolate mofetil in the prevention of rejection in de novo renal transplant recipients: a 3-year randomized, multicenter, phase III study. Transplantation. 2005;80:244-52

27. Vitko S, Margreiter R, Weimar W, Dantal J, Kuypers D, Winkler M, et al. Three-year efficacy and safety results from a study of everolimus versus mycophenolate mofetil in de novo renal transplant patients. Am J Transplant. 2005;5:2521-30

28. Tedesco-Silva H Jr, Vitko S, Pascual J, Eris J, Magee JC, Whelchel J, et al. 12-month safety and efficacy of everolimus with reduced exposure cyclosporine in de novo renal transplant recipients. Transpl Int. 2007;20:27-36

29. Tedesco-Silva H, Pascual J, Magee J, Civati G, Alves Filho G, Garcia V, et al. 36 months analysis on efficacy and safety of 2 doses of everolimus combined with reduced dose neoral $\mathbb{R}$ in de novo kidney transplant recipients. World Transplant Congress 2006 Poster Abstracts. Am J Transplant. 2006; 6 (s2), 473-1061 Abstract\# 1281

30. Whelchel J, Vitko S, Leone J, Eris J, Campbell S, Boubigott B, et al. 36 month result showing excellent graft function in de novo kidney transplant recipients treated with certican ${ }^{\circledR}, \operatorname{simulect}(\circledR$ and reduced neoral $(\mathbb{R}$ exposure. World Transplant Congress 2006 Oral Abstracts Am J Transplant. 2006; 6 (s2), 65-472 Abstract\# 695

31. Eisen HJ, Tuzcu EM, Dorent R, Kobashigawa J, Mancini D, Valantine-von Kaeppler HA, et al. Everolimus for the prevention of allograft rejection and vasculopathy in cardiac-transplant recipients. N Engl J Med. 2003;349:847-58

32. Radeva JI, Reed SD, Kaló Z, Kauf TL, Cantu E 3rd, Cretin N, et al. Economic evaluation of everolimus vs. azathioprine at one year after de novo heart transplantation. Clin Transplant. 2005;19:122-9

33. Holmes M, Chilcott J, Walters S, Whitby S, Akehurst R. Economic evaluation of everolimus versus mycophenolate mofetil in combination with cyclosporine and prednisolone in de novo renal transplant recipients. Transpl Int. 2004;17:182-7

34. Chapman JR, O'Connell PJ, Nankivell BJ. Chronic renal allograft dysfunction. J Am Soc Nephrol. 2005;16:3015-26. 